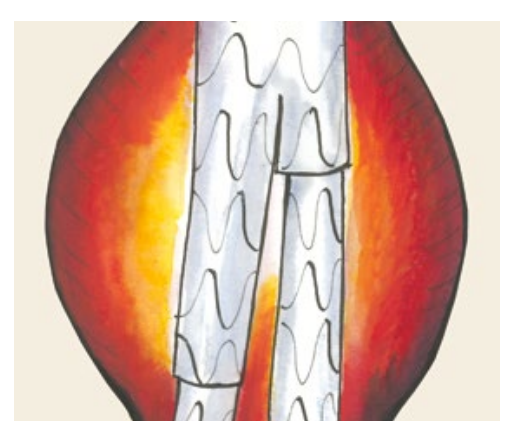

CPD

Jason Chuen, Mayo Theivendran

\section{Background}

Abdominal aortic aneurysm (AAA) remains one of the hallmark pathologies in vascular surgery and an area of intense research interest. Treatment options have expanded in recent years to increase the range of morphology suitable for endovascular aneurysm repair (EVAR), and with potential implications on treatment thresholds.

\section{Objectives}

This article is the first of two that will outline current treatment options for $A A A$, including areas of controversy and research in AAA disease, to inform the development of Australasian clinical guidelines and health policy.

\section{Discussion}

Medical therapy options remain limited and no aneurysm-specific pharmacotherapy is currently available. Recent years have witnessed a significant shift in AAA surgery from open repair to EVAR and expansion of EVAR techniques. General management of cardiovascular risk factors remains key to reducing all-cause mortality for patients with AAA.

\title{
Abdominal aortic aneurysm: An update
}

THE TREATMENT OF abdominal aortic aneurysm (AAA) has progressed significantly from the early days of open aneurysmorrhaphy and cellophane wrapping. The experiences of Albert Einstein and Rudolph Nissen now exist only in the annals of history, and the staple management of AAA disease includes targeted screening studies, patient education on detection, risk factor and medical management, screening for associated conditions, monitoring and surveillance until surgical treatment is warranted, and open repair or endovascular surgery followed by targeted postoperative surveillance.

This article outlines current medical and surgical treatment strategies, for the appreciation of practitioners managing patients with AAA. It is assumed that readers are familiar with the basic concepts described in our previous papers on aortic and non-aortic aneurysms. ${ }^{1,2}$

\section{Medical management of abdominal aortic aneurysm}

Medical management of AAA generally involves cardiovascular risk reduction, including antiplatelet therapy, statin therapy and antihypertensive therapy. This best medical management, however, is generally not intended to limit expansion or reduce the size of the AAA. Managing cardiovascular risk factors is crucial for improving the overall survival of patients and the outcomes of future AAA repair. A systematic review by Twine and Williams ${ }^{3}$ illustrated a significant all-cause mortality reduction at five years (odds ratio [OR]: 0.57) for those on statin therapy following AAA repair.

Smoking cessation should also be advised and encouraged in all patients with known AAA on the basis that it increases life expectancy and reduces morbidity and mortality from aneurysm repair. There is evidence that smoking is associated with AAA development and increased aneurysm expansion. ${ }^{4}$

Antihypertensive treatment should involve tailored blood pressure goals, although no specific agent has been shown to improve the outcome of AAA or provide postoperative benefit. Very few randomised trials to assess the effect of medicines on AAA growth rates in humans are available. The AARDVARK trial $^{5}$ assessing the effects of angiotensin converting enzyme (ACE) inhibitors on AAA growth has had disappointing results. A number of other trials such as the PISA trial, ${ }^{6}$ a study of the antiinflammatory effects of antihypertensive treatment in patients with small AAAs and mild hypertension, have been abandoned or terminated because of poor recruitment. In Australia, the Telmisartan in the management of abdominal aortic aneurysm (TEDY) trial assessing the effect of telmisartan is still in progress.

Diabetes has long been thought to be associated with a lower incidence of AAA. Review of large surveillance cohorts also suggest a lower expansion rate in patients with diabetes, ${ }^{8}$ most recently implicating the role of metformin. ${ }^{9}$

Studies continue to be performed on experimental animal models of AAA, and multiple reviews to date continue 
to identify areas of promise, but there remains no conclusive evidence that any pharmacological agent consistently and reliably modifies AAA expansion or rupture risk in a manner suitable for clinical use. ${ }^{10}$

\section{The role of surgery}

The past two decades have seen a significant shift in aortic aneurysm surgery, as indicated in the 2016 report from the International Consortium of Vascular Registries. ${ }^{11}$ This incorporated Australian and New Zealand data from the Australasian Vascular Audit ${ }^{12}$ and identified that, currently, 35-40\% of intact AAA repair was performed as open surgery. Significant variations have been seen, with $72 \%$ of intact AAA surgery being performed as open repair in Hungary, whereas only $21 \%$ of procedures were open repairs in the US.

Furthermore, the same report identifies that in some countries the threshold for AAA repair has reduced somewhat, leading to up to $31 \%$ of men and $12 \%$ of women being treated below traditionally accepted operative thresholds $(5.5 \mathrm{~cm}$ in men, $5.0 \mathrm{~cm}$ in women). ${ }^{13}$ This was associated with countries with higher use of EVAR and fee-for-service remuneration models, but significant variations were also seen within individual countries. In addition to the argument that endovascular aneurysm repair (EVAR) carries lower mortality and should be offered to patients at a lower rupture risk, proponents also point out that $61 \%$ of participants in the UK Small Aneurysm Trial (UKSAT) went on to have surgical repair because of aneurysm expansion, with an expected 4.5-5.5 cm growth period of only 2.3 years. ${ }^{14}$

Results from the PIVOTAL and CAESAR randomised trials confirm no benefit in treating patients with smaller AAA, and there remains no conclusive evidence to justify a reduction in guidelines for treatment threshold. Large registry analyses have demonstrated greater operative morbidity with very large $(>6.0 \mathrm{~cm})$ AAAs, ${ }^{15}$ but no benefit from treating if $<5.5 \mathrm{~cm}$ in diameter. A recent cost-effectiveness simulation analysis by Tomee et al found that reduction of the treatment threshold to $5.0 \mathrm{~cm}$ could result in significantly more surgical procedures, including re-interventions and subsequent operative mortality, at the cost of an estimated US $\$ 1$ million per rupture-related death prevented. ${ }^{16}$ The current guideline of $5.5 \mathrm{~cm}$ in men remains evidence-based practice, with scope for individualised risk evaluation on the basis of gender and family history, and earlier intervention on the basis of good clinical reasoning.

Australian national driving regulations stipulate that untreated atherosclerotic aortic aneurysms $>5.5 \mathrm{~cm}$ disqualify patients from an unconditional driver's licence except with the approval of a treating vascular surgeon. This is broadly consistent with clinical recommendations that aneurysms of that size should be treated. ${ }^{17}$

Current 2016 data $^{18}$ from the Australasian Vascular Audit (AVA) identify that $21.5 \%$ of elective AAA repairs were performed as open procedures, with an in-hospital mortality of $3.4 \%$, while $69 \%$ of ruptured AAA repairs were performed open, with an in-hospital mortality of $34.9 \%$. In comparison, the same data in $2010^{19}$ (the first full year of AVA reporting and 19 years after the first reported EVAR) showed $32 \%$ of elective AAA repairs as open, with $2.4 \%$ in-hospital mortality, and $75 \%$ of ruptured AAA performed open, with a $33 \%$ mortality. A review of publicly available Australian Medicare data for private sector surgery shows the change in treatment trends more dramatically (Figure 1).

\section{Endovascular aneurysm repair}

Endovascular surgery in the form of polyester or polytetrafluoroethylene bifurcated stent-graft aorto-iliac exclusion of the aneurysm sac has been widely accepted as an effective and viable method of treating AAAs, with the caveat that lifelong surveillance ${ }^{20}$ is required to observe for endoleak (ongoing filling of the residual aneurysm sac), graft migration or progressive aneurysmal change. While the first EVARs ${ }^{21-23}$ were performed in the Ukraine, Brazil and the United States, uptake and experience with EVAR in Australia and New Zealand has led the world through local development of stent-graft technologies. ${ }^{24}$

The outcomes after open and endovascular AAA repair have been well documented, and EVAR has a clear advantage with a lower, 30-day mortality over open repair. Large published cohorts from the American College of Surgeons National Surgical Quality Improvement Program (NSQIP) and US Medicare Benefits Schedule databases have

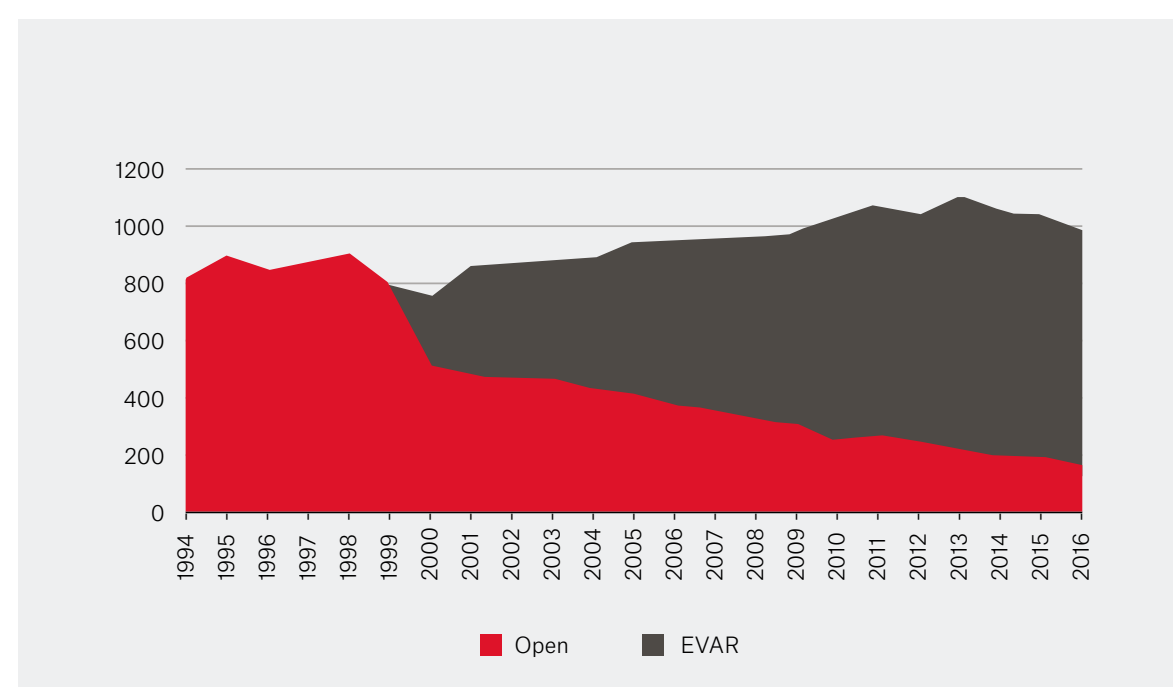

Figure 1. Medicare Benefits Schedule - AAA open versus EVAR procedures 
reported perioperative 30-day mortality of $1.3-1.7 \% .^{25,26}$

A systematic review of long-term data ${ }^{27}$ from early trials, such as EVAR 1 , DREAM, OVER and ACE, suggest that EVAR patients have slightly higher mortality and re-intervention rates, resulting in erosion of the early mortality benefits over the longer term. This finding is subject to the selection biases of prospective studies, the results from first-generation stent grafts, and improved appreciation of what requires re-intervention over the past 20 years of endovascular development.

The bane of EVAR is endoleak, graft occlusion and graft migration, which necessitate long-term surveillance and re-intervention. Patients with persistent endoleak, migration or graft infection may also require delayed open surgical revision and there is growing experience with operative techniques to handle this scenario.

Long-term data from the OVER trial found no significant difference in re-operation rates $(22.1 \% \mathrm{EVAR}$, compared with $17.8 \%$ open, $P=0.12),{ }^{28}$ while data from the UK EVAR 1 trial suggested an overall re-intervention rate of 4.1 interventions per 100 personyears after EVAR, compared with 1.7 for open repair, with the majority of these occurring in the first six months (hazard ratio [HR]: 2.37).${ }^{29} \mathrm{~A}$ more recent large cohort from the Veterans Affairs Surgical Quality Improvement Program (VASQIP) identified a higher re-intervention rate in open repair (10\%, compared with $6.3 \%$ after EVAR $)^{30}$ mostly due to hernia formation, adhesions and bowel obstruction.

Increasingly, EVAR has been offered as an option to borderline candidates. Female patients tend to have more aggressive aneurysmal growth, poor outcomes from rupture, and rupture earlier than men. Their iliac access vessels are smaller and hence were thought unsuitable for treatment with early generation EVAR grafts. Lower profile delivery systems that are now available permit easier negotiation of access vessels and low perioperative risk, so female patients can be treated earlier with good outcomes.
In patients with complex anatomies unsuitable for standard infrarenal endovascular bifurcate repair, a number of techniques have emerged to extend the suitability of endovascular treatment. These include fenestrated (FEVAR), branched (BrEVAR) and chimney (ChEVAR) grafts for thoracoabdominal repair, novel aneurysm neck sealing mechanisms (Anaconda, Ovation), supplementary embolisation of the residual aneurysm sac at the time of surgery, endovascular aneurysm sealing (EVAS) and, controversially, multilayer flow-modulating stents.

On the basis of the initial survival benefit, falling re-intervention rates due to stent graft design improvement, equivalent long-term survival to open repair and ever-expanding indication for use in complex anatomy, EVAR has become first-line treatment for infrarenal AAAs. Nevertheless, patients may opt for open repair to avoid the sometimes onerous long-term imaging and follow-up regimen, given its effective and durable history.

\section{Ruptured AAA}

Traditionally, it has been considered impractical to perform EVAR for a ruptured $\mathrm{AAA}^{31}$ because of the limited access to angiographic facilities and equipment for emergent EVAR, as well as the existing rapid access protocols for operating theatres in most vascular centres. Recent studies ${ }^{32,33}$ suggest that an emergency access protocol for emergency EVAR can yield acceptable results for treatment of ruptured AAA, assisted by rapid access to computed tomography (CT) and the use of intra-operative aortic balloon occlusion. Three-year results of the UK IMPROVE trial, ${ }^{34}$ randomising patients to an endovascular-first or open strategy in nominated aortic centres, shows improved survival and quality of life, equivalent re-intervention rates and reduced cost. Newly published practice guidelines from the US Society for Vascular Surgery now recommend EVAR as the preferred method of treatment if anatomically feasible, with a 'door to intervention' time of $<90$ minutes. ${ }^{13} \mathrm{Few}$ Australasian centres would currently be able to satisfy this target, and effective delivery will require installation of hybrid angiographic operating theatre facilities and revision of emergency protocols.

\section{Conclusions}

AAA disease continues to be a challenging and difficult condition to treat and much remains unknown about its biology and pathophysiology. This has hindered development of pharmacotherapy, and the mainstay of medical management remains treatment of known cardiovascular risk factors.

There is no strong, clinically viable human evidence that pharmacotherapy can slow or stop aneurysmal degeneration. Medical management can serve to improve cardiovascular outcomes and ensure optimisation prior to surgical intervention.

EVAR, through innovation in both device technology and clinical understanding, has proven to have good long-term outcomes comparable to open repair. Development of FEVAR, BrEVAR, EVAS and ChEVAR technology has given endovascular options with good clinical outcomes for patients with challenging anatomies. These techniques are increasingly acceptable alternatives to open surgery.

While progressive advances in endovascular technology will undoubtedly improve the deliverability and durability of this procedure, there is no suggestion that patients will be able to avoid longterm follow-up and regular investigations. In this setting, open repair remains a cornerstone of surgical therapy.

\footnotetext{
Authors

Jason Chuen MBBS, FRACS (Vasc), MPH, PGDipSurg Anat, Director of Vascular Surgery, Department of Vascular Surgery, Austin Health, Vic; Department of Surgery, Faculty of Medicine, Dentistry and Human Sciences, The University of Melbourne, Vic. jchuen@unimelb.edu.au

Mayo Theivendran MBBS, FRACS (Vascular), FRACS (General), Vascular, Endovascular and Transplant Surgeon, North Shore Private, Sydney, St Vincent's Private, Sydney, the Northern Beaches Hospital, Sydney, NSW, and Department of Vascular Surgery, Austin Health, Melbourne Vic Competing interests: None.

Provenance and peer review: Commissioned, externally peer reviewed.
} 


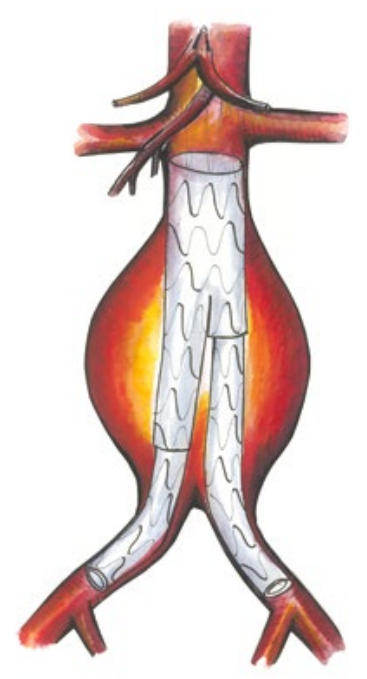

A

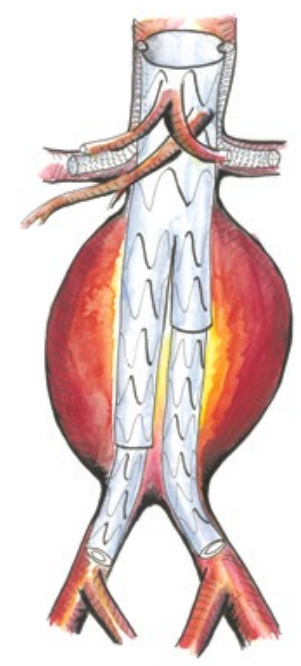

E

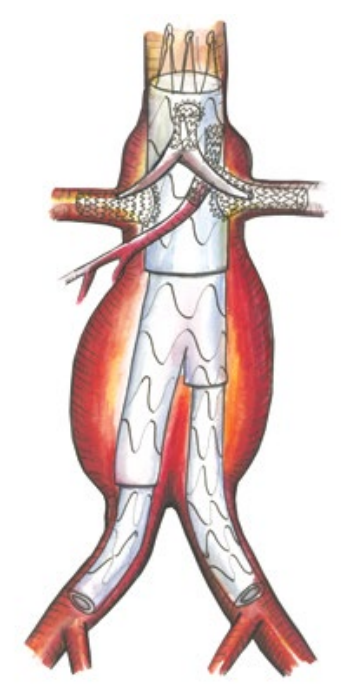

B

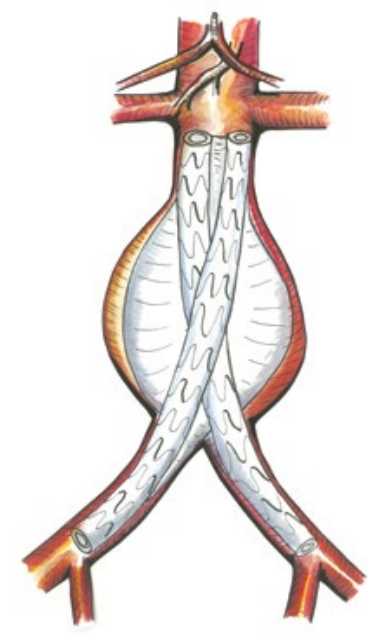

F
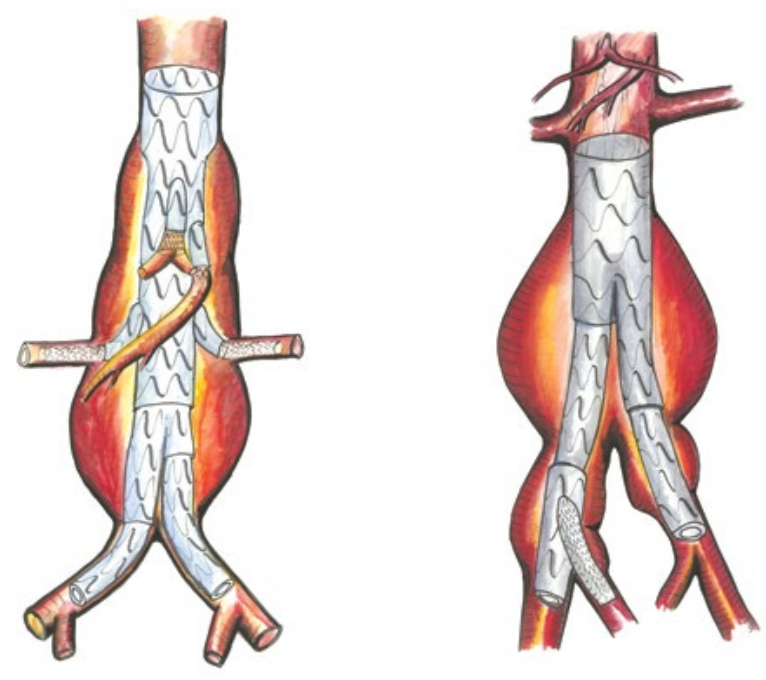

C

D

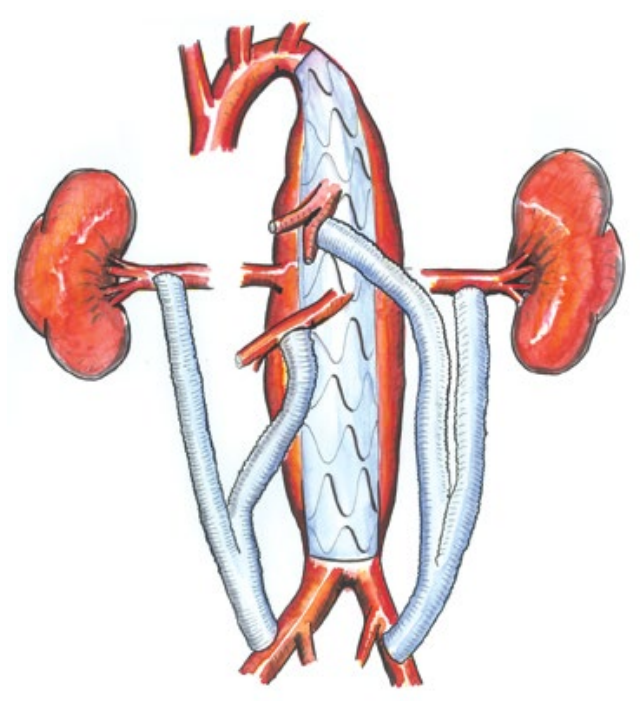

Figure 2. Types of endovascular repair (EVAR)

A. Standard EVAR, B. four-vessel fenestrated EVAR for thoracoabdominal aneurysm, C. four-vessel multibranch stent graft EVAR for thoracoabdominal aneurysm, D. Iliac side branch stent graft for aortoiliac aneurysm, E. two-vessel chimney EVAR for thoracoabdominal aneurysm, F. Nellix EVAS device, G. Hybrid thoracoabdominal debranching EVAR for thoracoabdominal or suprarenal aneurysm Illustrations by Dr Raf Ratinam

\section{References}

1. Robinson D, Mees B, Verhagen HJM, Chuen J. Aortic aneurysms - Screening, surveillance and referral. Aust Fam Physician 2013;42(6):364-69

2. Mees B, Robinson D, Verhagen HJM, Chuen J. Non-aortic aneurysms - Natural history and recommendations for referral and treatment. Aust Fam Physician 2013;42(6):370-74.
3. Twine CP, Williams IM. Systematic review and meta-analysis of the effects of statin therapy on abdominal aortic aneurysms. Br J Surg 2011;98(3):346-53. doi: 10.1002/bjs.7343.

4. Brady AR, Thompson SG, Fowkes FGR, Greenhalgh RM, Powell JT, UK Small Aneurysm Trial Participants. Abdominal aortic aneurysm expansion: Risk factors and time intervals for surveillance. Circulation 2004;110(1):16-21.
5. Bicknell CD, Kiru G, Falaschetti E, Powell JT, Poulter NR, AARDVARK Collaborators. An evaluation of the effect of an angiotensin-converting enzyme inhibitor on the growth rate of small abdominal aortic aneurysms: A randomized placebo-controlled trial (AARDVARK). Eur Heart J 2016;37(42):3213-21.

6. Blankensteijn JD. Study on anti-inflammatory effect of anti-hypertensive treatment in patients with small AAA's and mild hypertension (PISA). 
Bethesda, MD: U.S. National Library of Medicine: ClinicalTrials.gov, 2014. Available at https:// clinicaltrials.gov/ct2/show/NCT01425242 [Accessed 4 April 2018].

7. Morris DR, Cunningham MA, Ahimastos AA, et al. TElmisartan in the management of abDominal aortic aneurYsm (TEDY): The study protocol for a randomized controlled trial. Trials 2015;16(1):274. doi: 10.1186/s13063-015-0793-z.

8. Takagi H, Umemoto T, ALICE (All-Literature Investigation of Cardiovascular Evidence) Group. Diabetes and abdominal aortic aneurysm growth. Angiology 2016;67(6):513-25. doi: 10.1177/0003319715602414.

9. Golledge J, Moxon J, Pinchbeck J, et al. Association between metformin prescription and growth rates of abdominal aortic aneurysms. Br J Surg 2017;104(11):1486-93. doi: 10.1002/bjs.10587.

10. Golledge J, Norman PE, Murphy MP, Dalman RL. Challenges and opportunities in limiting abdominal aortic aneurysm growth. J Vasc Surg 2017;65(1):225-33. doi: 10.1016/j.jvs.2016.08.003.

11. Beck AW, Sedrakyan A, Mao J, et al. Variations in abdominal aortic aneurysm care: A report from the International Consortium of Vascular Registries. Circulation 2016;134(24):1948-58.

12. Bourke BM, Beiles CB, Thomson IA, Grigg MJ, Fitridge R. Development of the Australasian vascular surgical audit. J Vasc Surg 2012;55(1):164-69. doi: 10.1016/j.jvs.2011.06.102.

13. Chaikof EL, Dalman RL, Eskandari MK, et al. The Society for Vascular Surgery practice guidelines on the care of patients with an abdominal aortic aneurysm. J Vasc Surg 2018;67(1):2-77.e2. doi: 10.1016/j.jvs.2017.10.044.

14. Powell JT, Sweeting MJ, Brown LC, Gotensparre SM, Fowkes FG, Thompson SG. Systematic review and meta-analysis of growth rates of small abdominal aortic aneurysms. $\mathrm{Br} J$ Surg 2011;98(5):609-18. doi: 10.1002/bjs.7465.

15. Huang $Y$, Gloviczki P, Duncan AA, et al. Maximal aortic diameter affects outcome after endovascular repair of abdominal aortic aneurysms. J Vasc Surg 2017;65(5):1313-22. doi: 10.1016/j.jvs.2016.10.093.

16. Tomee SM, Bastiaannet E, Schermerhorn ML, Golledge J, Hamming JF, Lindeman JH. The consequences of real life practice of early abdominal aortic aneurysm repair: A cost-benefit analysis. Eur J Vasc Endovasc Surg 2017;54(1):2833. doi: 10.1016/j.ejvs.2017.03.025.

17. Austroads. Assessing fitness to drive for commercial and private vehicle drivers. 2 nd edn. Sydney: Austroads, 2017.

18. Australian \& New Zealand Society for Vascular Surgery. Australasian Vascular Audit Report, 2016. Melbourne: ANZSVS, 2017.

19. Australian \& New Zealand Society for Vascular Surgery. Inaugural Australasian Vascular Audit Report, 2010. Report no. 1. Melbourne: ANZSVS, 2011.

20. Cassagnes L, Pérignon R, Amokrane F, et al. Aortic stent-grafts: Endoleak surveillance. Diagn Interv Imaging 2016;97(1):19-27. doi: 10.1016/j. diii.2014.12.014.

21. Parodi JC, Palmaz JC, Barone HD. Transfemoral intraluminal graft implantation for abdominal aortic aneurysms. Ann Vasc Surg 1991;5(6):491-9.

22. Veith FJ, Marin ML, Cynamon J, Schonholz C, Parodi J. 1992: Parodi, Montefiore, and the first abdominal aortic aneurysm stent graft in the United States. Ann Vasc Surg 2005;19(5):749-51.

23. Volodos NL. Historical perspective: The first steps in endovascular aortic repair: How it all began. J Endovasc Ther 2013;20 Suppl 1:13-23. doi: 10.1583/1545-1550-20.sp1.I-3.
24. Lawrence-Brown MM, Hartley D, MacSweeney ST, et al. The Perth endoluminal bifurcated graft system - Development and early experience. Cardiovasc Surg 1996;4(6):706-12.

25. Malas M, Arhuidese I, Qazi U, Black J, Perler B, Freischlag JA. Perioperative mortality following repair of abdominal aortic aneurysms: Application of a randomized clinical trial to real-world practice using a validated nationwide data set. JAMA Surg 2014;149(12):1260-65. doi: 10.1001/ jamasurg.2014.275.

26. Schermerhorn ML, Giles KA, Sachs T, et al. Defining perioperative mortality after open and endovascular aortic aneurysm repair in the US Medicare population. J Am Coll Surg 2011;212(3):349-55. doi: 10.1016/j. jamcollsurg.2010.12.003.

27. Powell JT, Sweeting MJ, Ulug P, et al. Metaanalysis of individual-patient data from EVAR-1, DREAM, OVER and ACE trials comparing outcomes of endovascular or open repair for abdominal aortic aneurysm over 5 years. Br J Surg 2017 Feb;104(3):166-78. doi: 10.1002/bjs.10430.

28. Lederle FA, Freischlag JA, Kyriakides TC, et al. Long-term comparison of endovascular and open repair of abdominal aortic aneurysm. N Engl J Med 2012;367(21):1988-97. doi: 10.1056/ NEJMoa1207481.

29. Patel R, Sweeting MJ, Powell JT, Greenhalgh RM, EVAR trial investigators. Endovascular versus open repair of abdominal aortic aneurysm in 15-years' follow-up of the UK endovascular aneurysm repair trial 1 (EVAR trial 1): A randomised controlled trial. Lancet 2016;388(10058):2366-74. doi: 10.1016/ S0140-6736(16)31135-7.

30. Hynes CF, Endicott KM, Iranmanesh S, Amdur RL, Macsata R. Reoperation rates after open and endovascular abdominal aortic aneurysm repairs. J Vasc Surg 2017;65(5):1323-28. doi: 10.1016/j. jvs.2016.09.053.

31. Lo J, Jedynak J, Chuen J. Perceived barriers to endovascular repair of ruptured abdominal aortic aneurysm among Australasian vascular surgeons. J Vasc Surg 2016;64(2):328-32. doi: 10.1016/j. jvs.2016.01.037.

32. IMPROVE Trial Investigators. Endovascular strategy or open repair for ruptured abdominal aortic aneurysm: One-year outcomes from the IMPROVE randomized trial. Eur Heart J 2015;36(31):2061-69.

33. Oyague KS, Mubarak OA, Nowak LR, et al. Endovascular repair of ruptured and symptomatic abdominal aortic aneurysms using a structured protocol in a community teaching hospital. Ann Vasc Surg 2015;29(1):76-83.

34. IMPROVE Trial Investigators. Comparative clinical effectiveness and cost effectiveness of endovascular strategy $v$ open repair for ruptured abdominal aortic aneurysm: Three year results of the IMPROVE randomised trial. BMJ 2017;359:j4859. doi: 10.1136/bmj.j4859. 